Harald Meden

Am Freitag, den 7. Juni 2013, fand in der historischen Hörsaalruine der Charité in Berlin eine Jubiläumsveranstaltung der wissenschaftlichen Fachgesellschaft NATUM statt. NATUM steht für Naturheilkunde, Komplementärmedizin, Akupunktur und Umweltmedizin in der Frauenheilkunde. Die NATUM ist eine wissenschaftliche Arbeitsgemeinschaft der Deutschen Gesellschaft für Gynäkologie und Geburtshilfe (DGGG e.V.). Sie ist eine Arbeitsgemeinschaft ganzheitlich denkender und naturheilkundlich tätiger Ärzte und Wissenschaftler. Gegründet wurde die NATUM 1993 von Frau Professor Ingrid Gerhard, der damaligen Leiterin der Ambulanz für Naturheilkunde der Universitätsfrauenklinik, in Heidelberg.

Insbesondere im Bereich der Onkologie hat sich die NATUM seit ihrem Bestehen in besonderer Weise wissenschaftlich betätigt und wesentlich zur Akzeptanz ergänzender Massnahmen in der Onkologie beigetragen, sodass diese inzwischen an vielen Kliniken und Universitäten Eingang in die Routine gefunden haben.

Die NATUM hat zur Konzentrierung verschiedener naturheilkundlicher Verfahren eigene Arbeitsgemeinschaften auf freiwilliger Interes-

\title{
20 Jahre wissenschaftliche Fachgesellschaft NATUM
}

Abb. 1.

Die Preisträgerin Frau Professor Dr. med. Sigrun Chrubasik (Mitte) sowie der Vorstand der NATUM.

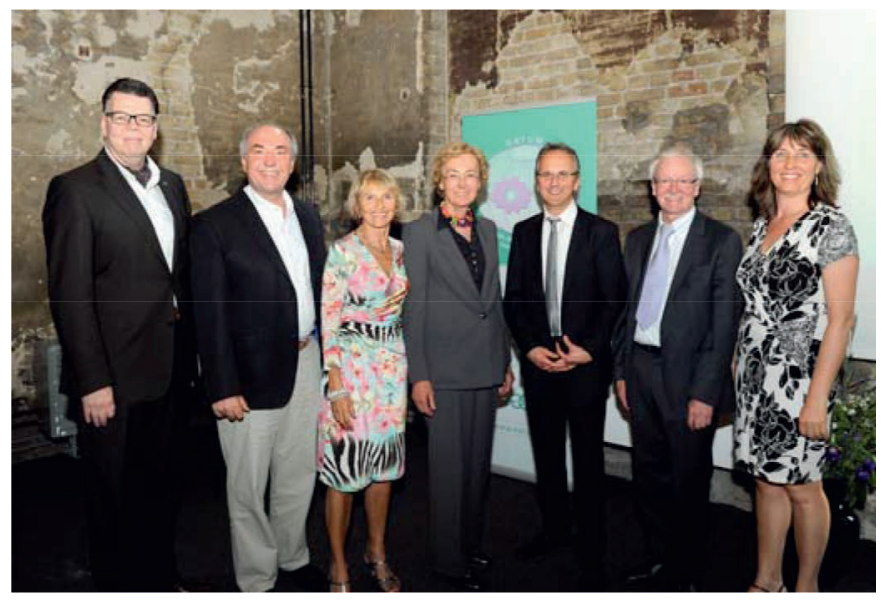

senbasis eingerichtet. Die NATUM führt Studien durch bzw. unterstützt diese und hat diverse universitäre Kooperationen, um Forschung, Lehre und Leitlinien im Bereich der Komplementärmedizin und sonstiger $\mathrm{Na}-$ turheilverfahren zu fördern.

$\mathrm{Zu}$ dieser Förderung gehört die Verleihung des diesjährigen Wissenschaftspreises, der mit 3000 Euro dotiert ist. Dieser Wissenschaftspreis wurde im Rahmen der jetzigen akademischen Feier an Frau Professor Dr. med. Sigrun Chrubasik für ihre Arbeiten auf dem Gebiet der Naturheilverfahren verliehen.

Die Laudatio wurde von Frau Professor Ingrid Gerhard gehalten und der Festvortrag von Prof. Dr. Andreas Michalsen vom Immanuel Krankenhaus in Berlin. Das Grusswort des Berufsverbandes der Frauenärzte wurde von ihrem Präsidenten, Dr. Christian Albring, gesprochen. Beiträge früherer und heutiger Vorstandsmitglieder der NATUM zur Gründung und weiteren Entwicklung sowie eine musikalische Umrahmung durch das Aloysia Quartett rundeten das Programm ab.

Begleitend erfolgte die Besichtigung des Medizinhistorischen $\mathrm{Mu}-$ seums der Charité mit der Dauerausstellung «Dem Leben auf der Spur» zu 300 Jahren Medizingeschichte. Weitere Informationen finden Sie unter www.natum.de. 